\title{
Entre les bons et les méchants, entre le texte et la danse : La belle au bois dormant selon Perrault, Petipa, Ek et Lock
}

\section{Sarah ANTHONY, Université McGill}

Il est indéniable que l'art de la danse classique et celui de la littérature sont fortement liés. Roméo et Juliette, Don Quichotte, Cendrillon et Carmen ne sont que quelques exemples de ballets qui puisent leur inspiration dans des d'œuvres littéraires. Ainsi, il est peu surprenant qu'en 1890, Marius Petipa mette en scène La belle au bois dormant qui suit les grandes lignes du conte de Charles Perrault, d'autant plus que les contes de fées, institutionnalisés depuis le dix-septième siècle, étaient devenus des prototypes narratifs pour la civilisation occidentale (Canton 17). Mais comment s'effectue ce passage du texte à la danse ? Quels sont les mécanismes de cette transposition intermédiale? Au cours de nos recherches, nous avons relevé quatre procédés textuels - la reconfiguration, la substitution, l'élimination et l'addition - qui ont permis au librettiste Ivan Vsevolozhsky, au compositeur Piotr Ilitch Tchaïkovski et au chorégraphe Petipa d'adapter le texte de Perrault afin de créer ce qu'on appelait à l'époque un ballet-féerie ${ }^{1}$. En poursuivant cette réflexion, nous avons également découvert que les archétypes ${ }^{2}$ du bien et du mal sont des outils importants pour la transposition du textuel au gestuel dans La belle au bois dormant de Petipa. Dans cette étude, nous élargirons notre corpus pour voir comment Mats Ek et Édouard Lock, des chorégraphes contemporains, utilisent ce même outil cognitif dans leurs réinterprétations du ballet classique de Petipa, soit Sleeping Beauty (1996) et Amjad (2007) respectivement. En prenant comme exemple La belle au bois dormant, ce travail visera donc à mieux cerner ce qui se passe lorsqu'un texte est mis en mouvement, en accentuant notamment l'importance de l'archétype pour les rapports entre le conte, le ballet classique et les adaptations contemporaines. En examinant les emplois que font trois chorégraphes de l'archétype, nous chercherons aussi à établir si l'usage de cet outil de transposition change d'une époque à une autre, de la période du ballet classique (Petipa) à l'ère du ballet contemporain (Ek et Lock).

\section{Du conte au libretto : une dichotomie archétypale en relief}

Dans une communication donnée en 2008, nous avons constaté que les procédés de la reconfiguration, de la substitution, de l'élimination et de l'addition étaient employés dans le 
libretto de La belle au bois dormant pour rendre aussi claire que possible la distinction entre les personnages bienveillants et malveillants sur scène, une différentiation qui est un élément essentiel à la mise en mouvement du texte de Perrault. Cette dichotomie archétypale, entre le bien et le mal, a longtemps été perpétuée, entre autres, par le christianisme et, en raison de cela, ce concept s'avère aisément reconnaissable pour un vaste public. De ce fait, Vsevolozhsky, le librettiste, porte une partie importante de ses efforts éditoriaux, peut-être de façon instinctive, sur la mise en valeur de cette dichotomie pour permettre ensuite à Petipa et à Tchaïkovski de transposer le conte de fées sur scène, afin qu'il soit compris dans son ensemble par la majorité des spectateurs.

Le contraste qu'établit Vsevolozhsky entre les bons et les méchants, est accentué par la manière selon laquelle il reconfigure la chronologie des événements de l'histoire de Perrault. Dans le conte, la vieille fée arrive sans avoir été invitée au baptême au moment où l'on se mettait à table, c'est-à-dire bien avant la cérémonie des dons, pendant laquelle chaque fée allait offrir sa bénédiction à la princesse. Par contraste, dans le libretto, Carabosse surgit et interrompt la Fée des Lilas qui se penche pour présenter son don à la princesse Aurore. Cette réorganisation de la chronologie des événements sert non seulement à créer un moment dramatique au sein du spectacle, mais elle indique surtout aux membres de l'auditoire que la fée Carabosse est l'opposante principale d'Aurore, et que la Fée des Lilas est l'adjuvante la plus importante de la jeune princesse.

Qui plus est, le librettiste substitue certains personnages à d'autres et élimine presque le tiers des péripéties de l'hypotexte, de sorte à concentrer la malveillance tout entière en une seule figure : celle de la méchante fée Carabosse. Ainsi, la fileuse qui, dans le conte de Perrault, donne à la princesse le fuseau avec lequel elle se piquera, est remplacée dans le libretto par la méchante fée Carabosse, qui se déguise en vieille dame, assurant la réalisation du mauvais sort qu'elle avait jeté sur la princesse. Vsevolozhsky supprime également tous les événements du conte qui viennent à la suite du mariage du prince et de la princesse. Ceux-ci renvoient principalement aux penchants cannibales de la mère du prince qui était une ogresse. Évidemment, l'épisode du carnage présent dans l'hypotexte - pendant lequel l'ogresse demande à son maître d'hôtel de lui servir ses deux petits-enfants et sa bru dans une sauce-robert - se serait mal prêté à l'esthétique du ballet-féerie qui devait être spectaculaire et non pas morbide. En outre, en remplaçant cette partie de l'histoire avec les festivités du mariage, éliminant de la sorte l'ogresse, le librettiste peut se permettre de donner plus d'importance à la méchante fée Carabosse, qui occupe un rôle plutôt secondaire dans 
le conte de Perrault. Sa fée Carabosse remplace donc toutes les personnifications du mal que l'on retrouve dans le conte, soit la vieille fée et l'ogresse ainsi que la fileuse qui participe inconsciemment à la réalisation du mauvais sort.

Vsevolozhsky emploie aussi le procédé de l'addition lorsqu'il décrit la fée qu'on avait oublié d'inviter à la cérémonie du baptême qui est, par contraste, dépeinte très succinctement dans l'extrait suivant du conte de Perrault :

Mais comme chacun prenait sa place à table, on vit entrer une vieille Fée qu'on n'avait point priée parce qu'il y avait plus de cinquante ans qu'elle n'était sortie d'une Tour et qu'on la croyait morte ou enchantée. Le Roi lui fit donner un couvert, mais il n'y eut pas le moyen de lui donner un étui d'or massif, comme aux autres, parce que l'on n'en avait fait faire que sept pour les sept Fées. La vieille crut qu'on la méprisait, et grommela quelques menaces entre ses dents. [...] Le rang de la vieille Fée étant venu, elle dit, en branlant la tête encore plus de dépit que de vieillesse, que la Princesse se percerait la main d'un fuseau, et qu'elle en mourrait. Ce terrible don fit frémir toute la compagnie, et il n'y eut personne qui ne pleurât. (97-98; nous soulignons)

En présentant la fée qui n'avait pas été invitée au baptême comme asociale et rancunière, Perrault concrétise le concept abstrait du mal, tel que personnifié par cette fée, au moyen de ces deux caractéristiques. Il crée ainsi une représentation de la notion archétypale du mal qui s'avère beaucoup moins étoffée et très différente de celle que décrit Vsevolozhsky dans son libretto, dont en voici un extrait de la traduction anglaise :

When her turn comes the Lilac Fairy approaches the cradle to present her gift, as suddenly a loud noise is heard at the entrance ; a page runs in and informs Catalabutte [master of ceremonies] that a new fairy, whom they forgot to invite to the feast, is already at the castle gate. This is the evil fairy Carabosse - the most powerful and evil in the entire land. [...] Carabosse appears in a wheelbarrow drawn by six large rats ; ugly dirty pages accompany her. [...] Catalabutte, neither dead nor alive, throws himself at the knees of the evil fairy, begging her for forgiveness and promising to serve her faithfully to the end of his days. Carabosse laughs at him mockingly and entertains herself by plucking out tufts of his hair and throwing them at the rats, who devour them. Soon Catalabutte's head is completely bald. [...] « Aurora, thanks to the gifts of her six godmothers », says Carabosse, "will be the most beautiful, the most seductive, the most clever of all the princesses in the world. I do not have the power to deprive her of these qualities, but in order that her happiness never be disturbed - you see how good I am - she will fall asleep the first time she pricks her finger or hand, and her slumber will be forever ». The King, Queen, and entire court are dumbfounded. Carabosse makes signs with her wand over the cradle, pronouncing magic words and, happy at the trick she has played on her sisters, begins to guffaw; her gaiety is transferred to all her ugly retinue. (Wiley 327-328; nous soulignons) 
Dans le libretto, à la différence du conte, la malfaisance n'est pas tout simplement impliquée par le biais d'un caractère asocial et rancunier, elle est rendue explicite, entre autres, par l'emploi de l'adjectif « evil », qui est accompagné du superlatif «the most ». Vsevolozhsky utilise également des moyens plus subtils pour insister sur le fait que cette fée est une personnification, voire une concrétisation du concept archétypal du mal. Il lui attribue le nom de «Carabosse », qui renvoie à un type de difformité physique, caractéristique qui sert souvent à dénoter le mal, comme l'accentue Marie-Louise von Franz dans son ouvrage Shadow and Evil in Fairy Tales : « in the archetypal experience of evil, evil powers are seen as a crippled human, or as a distorted thing » (179).

Dans le texte de Vsevolozhsky, la représentation du mal, telle que personnifiée par la fée Carabosse, compte également trois autres éléments, de nature sonore, visuelle et comportementale, qui n'apparaissent pas dans l'hypotexte. Premièrement, l'arrivée de la fée Carabosse est marquée d'un son fort, ce qui perturbe l'harmonie des airs qui accompagnaient la scène des bénédictions. Deuxièmement, la brouette de la fée Carabosse est tirée par des rats, symboles de saleté et de maladie $^{3}$, et elle est escortée par des pages qui sont dépeints comme étant sales et laids. Enfin, à la différence de la vieille fée du conte de Perrault, qui ne fait que jeter par dépit un mauvais sort sur la princesse, les actions malveillantes de Carabosse sont nombreuses. En plus du mauvais sort qu'elle jette sur la princesse, elle punit Catalabutte en lui arrachant les cheveux, elle se déguisera en vieille dame pour donner le fuseau fatidique à Aurore, et son rire, qui est décrit par le librettiste comme étant diabolique, ponctue la plupart de ses mauvaises actions. Par ailleurs, Vsevolozhsky utilise aussi le processus de l'addition dans la représentation qu'il fait de la Fée des Lilas, dans le sens où son rôle est plus important dans le libretto que dans le conte. Par exemple, dans le texte de Perrault, c'est la curiosité du Prince qui l'amène à explorer le château ensorcelé, tandis que dans le libretto, c'est la Fée des Lilas qui le guide vers la princesse endormie. La fée bienveillante fait donc preuve d'une présence plus marquée dans le libretto et, par extension, dans le ballet classique.

Les remaniements apportés au conte par le librettiste indiquent qu'il fait face à au moins deux contraintes. Premièrement, son libretto doit faciliter le travail d'adaptation que feront par la suite Petipa et Tchaïkovski. De ce fait, une grande majorité des modifications - l'ajout des éléments visuels (les rats et les pages), sonores (le bruit fort qui marque l'arrivée de Carabosse, son rire diabolique) et comportementaux (les diverses actions malveillantes attribuées à Carabosse) - qu'il apporte au conte de Perrault se prêtent autant à un texte (le libretto) qu'à sa mise en scène. Deuxièmement, comme librettiste, il cherche à s'assurer que l'histoire transposée soit aussi 
compréhensible que possible, tant dans le libretto que dans la pièce dansée. Pour ce faire, lorsqu'il s'agit de mettre en relief les archétypes du bien et du mal, Vsevolozhsky donne un rôle plus important à la Fée des Lilas et il unifie les multiples personnifications que fait Perrault du mal en un personnage, tout en amplifiant et explicitant la malveillance de la vieille fée qui est plutôt implicite dans le conte. De la sorte, il tâche de garantir que la fée bienveillante et son opposante seront perçues par la majorité des spectateurs comme des personnifications du bien et du mal, facilitant ainsi, en partie, la mise en mouvement du conte.

\section{Du libretto à la scène : donner corps aux archétypes}

En comparant le libretto à une représentation du ballet de Petipa, nous avons remarqué que même si Vsevolozhsky s'évertue à contrôler, entre autres, la perception qu'auront les spectateurs des personnages de Carabosse et de la Fée des Lilas, il donne toutefois une certaine liberté créatrice à Petipa quant à la représentation visuelle qu'il fera de ces fées, puisque le libretto ne donne pas de description physique de celles-ci. En examinant la scène de la malédiction de Carabosse telle que dansée par le Kirov Ballet en 1982, on constate que tout comme Vsevolozhsky, Petipa accentue à sa façon la dichotomie archétypale du bien et du mal, notamment au moyen de la gestuelle qu'il donne à ses danseurs. Accompagnés d'une musique violente et diabolique, les mouvements de la fée Carabosse se distinguent de ceux qu'emploient les autres personnages du ballet par leur qualité abrupte, inélégante et spasmodique. Typiquement, les mouvements qu'exécutent les danseurs de ballet sont très stylisés ; ils sont gracieux, élégants, légers et précis. C'est en établissant un contraste entre la gestuelle typique et les mouvements atypiques que Petipa fait la différence entre les bons et les méchants sur scène. De même, les couleurs des costumes et le ton de la musique aident à discerner les personnages bienveillants de ceux qui ne le sont pas. Dans cette séquence, les couleurs sombres que portent la fée Carabosse et sa suite, s'opposent aux couleurs claires des six bonnes fées, d'autant plus que Carabosse et la Fée des Lilas ont chacune leur propre leitmotiv musical qui les caractérise et les rend reconnaissables par l'ouïe. Ces détails ne font pas partie du libretto et constituent les éléments ajoutés au scénario par la mise en scène quant à l'évocation gestuelle, visuelle et auditive de la dichotomie archétypale du bien et du mal.

Afin de développer sa propre représentation de la notion archétypale du mal, Petipa a également recours au travestissement. Même si la grammaire du conte et celle du libretto indiquaient que la méchante fée devrait être une femme, ce fut le célèbre danseur Enrico Cecchetti 
qui dansa le rôle de la fée Carabosse lors de la première en 1890. À l'époque, le travestissement était une pratique courante dans le domaine du ballet. Petipa n'était pas le seul à employer cette stratégie pour accentuer la malveillance d'un personnage ; notamment, le rôle de la méchante fée Madge dans La sylphide, un ballet du chorégraphe danois August Bournonville, fut dansé pour la première fois en 1836 par un homme, Carl Fredstrup. Il nous semble que, dans les deux cas, l'ambiguïté sexuelle de l'homme en travesti servait à insister sur la monstruosité du personnage en question. Ni femme, ni homme, les personnages de Carabosse et Madge étaient conçus pour évoquer un sentiment d'anxiété chez le spectateur, qui devait en principe ressentir une certaine abjection à l'égard de ces méchantes fées en raison de leur apparence troublante. En outre, à l'époque, le public avait une attitude plutôt négative envers le ballerin, qui se voit éclipsé par le culte de la beauté féminine qui a marqué l'âge romantique du ballet (de 1830 jusqu'à la fin du dixneuvième siècle). C'est pendant cette période que l'on a introduit les tutus et les pointes dont les bouts durs permettaient aux ballerines de produire une illusion d'extrême légèreté.

Se prêtant moins bien à cette nouvelle esthétique, le ballerin ${ }^{4}$ passe ainsi au second plan ; il devient le partenaire qui soulève la danseuse et met en valeur sa grâce. Par rapport aux rôles créés pour les ballerines, hormis quelques exceptions, ceux attribués à l'époque aux danseurs étaient secondaires, moins «dansés » et plutôt mimés, comme fut celui de la fée Carabosse. L'effacement du danseur au cours du dix-neuvième siècle s'explique également, selon Ramsay Burt et Michael Gard, par des raisons socio-économiques de l'époque, tout comme l'atteste cet extrait de l'excellent ouvrage de ce dernier intitulé Men Who Dance : Aesthetics, Athletics and the Art of Masculinity:

First, this period was marked by [...] middle class anxiety about gender relations within a rapidly industrialising and urbanising society. [...] Second, outside the art form bourgeois society seems to have associated ballet with an effete aristocratic sensibility, which positioned the male dancer in opposition to the new professional, productive and soberly attired male body. This coincides with a lowering of the status of ballet generally, a factor which is tied up with its association with the female body and which made it an unlikely career choice for men of any social strata. (51)

Quant à la place du danseur dans l'esthétique du ballet romantique, Gard signale que « the dancing male body came to be an unwanted reminder of the impure, fecund and overly vigorous working class body » (51). Autrement dit, le corps du danseur représentait une menace envers la pureté d'une beauté féminine idéalisée. En fin de compte, le ballerin est devenu en quelque sorte le 
souffre-douleur de cette époque marquée par des malaises sociaux. Tenant compte des raisons susmentionnées, nous proposons que l'homme en travesti ait suscité encore plus d'anxiété chez les spectateurs que le danseur non déguisé, se prêtant ainsi particulièrement bien à l'évocation de la malveillance.

\section{De Petipa à Ek : vers un classique actualisé}

Un siècle plus tard, en 1996, le danseur et chorégraphe suédois Mats Ek revient sur l'histoire de La belle au bois dormant. Dans sa reprise intitulée Sleeping Beauty, Ek modifie complètement les événements du conte et développe un nouveau récit au moyen d'une gestuelle à la fois classique, contemporaine et folklorique. Hormis quelques remaniements quant à l'ordre des pièces musicales, seule la musique de Tchaïkovski reste plus ou moins inchangée. Ek, un des enfants terribles de la danse contemporaine, rend le conte de Perrault et le ballet-féerie de Petipa presque méconnaissables. C'est d'ailleurs ce qu'il a fait avec une variété de ballets classiques dont Giselle, Le lac des cygnes et Carmen - des adaptations qui, par leur humour noir et leur créativité, lui ont assuré une renommée mondiale. Chez Ek, Sleeping Beauty devient donc très sensuel, violent et psychologique, une version du conte de fées qui est destinée aux adultes plutôt qu'aux enfants. Pour commencer, cette chorégraphie met en scène l'amour que partagent la reine Silvia et le roi Florestan ainsi que leur désir d'avoir un enfant, des détails qui ne figurent ni dans le conte ni dans le ballet-féerie. Ensuite, la cérémonie des dons est remplacée par la naissance d'Aurore à l'hôpital, où la reine Silvia est accompagnée par les bonnes fées et soignée par le médecin, Carabosse, qui facilite son accouchement grâce à des analgésiques. Par la suite, on découvre qu'Aurore est devenue une jeune fille gâtée, fugueuse et de mœurs légères, qui finit par tomber amoureuse de Carabosse, qui réapparaît sur scène comme un jeune immigrant. Ce dernier initie Aurore à l'héroïne, il entretient ensuite un rapport abusif avec elle et, finalement, il est tué par le Prince Désiré. Aurore se voit enfin libérée de sa toxicomanie et elle se marie avec son prince charmant, comme dans le conte de fées. Malheureusement, elle donne naissance à un enfant dont le père, on s'en doutait bien, n'est pas le Prince Désiré, mais plutôt Carabosse. Nous voilà donc au sein d'un récit aux nuances contemporaines qui ne retient que quelques vestiges du conte qui l'a inspiré.

En examinant l'extrait du Sleeping Beauty d'Ek, dansé par le Cullberg Ballet ${ }^{5}$ en 1999, qui correspond musicalement à la scène de la malédiction de la fée Carabosse dans La belle au bois 
dormant de Petipa, on se retrouve à l'hôpital au moment où la reine Silvia accouche d'Aurore. Dans cette séquence, comme dans bien d'autres d'ailleurs, Ek ne suit pas les règles du ballet classique. Notamment, il met en scène des épisodes - comme celui de la naissance d'Aurore - qui sont trop vulgaires pour le style du ballet-féerie qui, rappelons-le, cherche à être spectaculaire et beau. En outre, à plusieurs reprises, la chorégraphie est beaucoup trop sensuelle et violente pour ce type de spectacle; les danseurs miment des actes sexuels, Aurore et Carabosse s'injectent de drogues fortes et le Prince Désiré tue Carabosse en le criblant de balles. De toute évidence, la majorité des costumes choisis ne respectent pas l'esthétique du ballet-féerie, qui cultivait la féminité et la légèreté. Pour ne citer qu'un exemple, dans la scène en question, la Fée des Lilas porte un pantalon et des souliers de ballet, plutôt qu'un tutu et des pointes. La contemporanéité de ce ballet est aussi soulignée par la présence de certains objets sur scène, comme les télévisions, le téléphone et la voiture, qui semblent mettre en relief la culture de consommation qui était bien présente dans les années quatre-vingt-dix et qui persiste aujourd'hui. Les ajouts que fait Ek à l'histoire dansée bien connue de La belle au bois dormant, visent donc à accentuer plusieurs préoccupations de son époque comme l'obsession du plaisir sexuel, l'accumulation de biens et la toxicomanie. Comme l'ont remarqué bien des critiques, tels que Mårten Spångberg (1996), Gunilla Jensen (1997) et Vida Midgelow (1999), la tendance qu'a cet artiste suédois à actualiser les classiques de cette façon, est une caractéristique de son style chorégraphique.

D'ailleurs, les représentations qu'il fait du bien et du mal dans Sleeping Beauty, sont renouvelées par cette mise en valeur des angoisses sociales de son époque qui est si importante pour ses œuvres. De ce fait, pour insister sur sa malveillance, le personnage de Carabosse est un étranger, ce qui est suggéré par l'attribution de ce rôle à un danseur égyptien, Gamal Gouda, dont la physionomie est en contraste avec celle d'Aurore - une blonde aux yeux bleus - qui est typiquement scandinave. Et si le choix des danseurs ne rendait pas évidente «l'étrangeté » de Carabosse, les propos du Prince Désiré qui le traite de «Goddam immigrant» rendent ceci explicite. Ce n'est plus le travestissement qui sert à souligner la monstruosité du personnage de Carabosse, mais plutôt la couleur de sa peau. La xénophobie qu'exprime le Prince Désiré - que le chorégraphe veut certainement dénoncer - fait écho au sentiment de dégoût qu'on devrait avoir envers Carabosse en raison de sa malveillance. De plus, l'antipathie de ce personnage est également accentuée du fait qu'il est héroïnomane et qu'il violente Aurore ainsi qu'une vieille dame qu'il cherche à dévaliser. Il importe aussi de souligner que dans le ballet classique, les effets 
de la malveillance de Carabosse sont d'abord mitigés par le don de la Fée des Lilas et ensuite complètement effacés par le baiser du Prince Désiré. Dans le Sleeping Beauty d'Ek, la rédemption d'Aurore lorsqu'elle se marie avec son prince n'est que temporaire, car elle donne naissance au bébé de Carabosse. Par contraste au ballet-féerie, où le mal est graduellement éradiqué par le bien, dans le ballet contemporain d'Ek le rideau tombe sans que le mal ait disparu. Certes, ces différences entre la relation que partagent les notions archétypales du bien et du mal chez Ek et chez Petipa, reflètent une divergence d'esthétiques, mais elles découlent aussi du fait que chaque chorégraphe fait face à des contraintes distinctes. Rappelons qu'en 1890, les archétypes sont employés par Petipa pour raconter une histoire méconnue, donc la représentation du rapport entre le bien et le mal doit être aussi claire que possible, une force effaçant progressivement l'autre. En 1996, l'histoire d'Aurore est familière, alors le chorégraphe peut se permettre de complexifier le lien entre les deux entités pour montrer que les rapports humains et les individus qui y participent ne sont pas unidimensionnels.

Il est intéressant de remarquer que le travestissement n'est pas utilisé par Ek pour créer une angoisse chez le spectateur. Ceci peut s'expliquer du fait que la tension que génère la présence du ballerin sur scène au dix-neuvième siècle s'est presque complètement dissipée vers la fin du vingtième siècle. Par conséquent, le travestissement est souvent employé dans les ballets contemporains à des fins parodiques et burlesques. Nous pensons notamment aux Ballets Trockadero de Monte Carlo, troupe américaine fondée en 1974, qui met en scène des ballets classiques dansés en travesti par des hommes avec des résultats hilarants. Vu qu'il est évident qu'Ek cherche à déconstruire les ballets classiques, c'est logique qu'il ne perpétue pas la tradition du travestissement dans sa réinterprétation de ce type de production. D'ailleurs, ce n'est pas la seule convention relative au genre qui est transformée par Ek. Par contraste à la gestuelle et à la structure des ballets-féeries, qui sont grandement influencées par une hiérarchie entre homme et femme où ce premier est éclipsé par cette dernière, l'œuvre du chorégraphe suédois est surtout composée de mouvements qui ne sont ni féminins, ni masculins et elle ne comporte aucun pas de deux proprement dit où la femme est mise en valeur. Midgelow a donc raison de dire que la gestuelle d'Ek « is not a gender specific one. The movement language usefully opens up gender binaries leading to much more contemporary representations of men and women. Ek exposes complexities of gender by inverting the far more conventional, stereotyped and idealised figures of traditional ballet » (7). 


\section{De Petipa à Lock : souvenirs évoqués et classiques déconstruits}

Dans Amjad (2007), Édouard Lock joue lui aussi avec les conventions qui régissent les rapports entre hommes et femmes. De renommée mondiale, sa troupe montréalaise La La La Human Steps $^{6}$ a développé, au cours des années, une gestuelle complexe, à la fois frénétique et provocatrice, qui est presque indissociable de l'incomparable Louise Lecavalier, l'interprète musclée à la chevelure platine rebelle, qui a longtemps servi de muse à Lock, interprétant la chorégraphie de celui-ci sans aucune retenue apparente. Quoique le répertoire de La La La Human Steps ne compte qu'une dizaine de pièces, celles-ci sont en revanche presque débordantes de mouvement et une de ses dernières créations, Amjad, ne fait pas exception à la règle. Sans entracte, ce ballet contemporain de quatre-vingts minutes renvoie aux classiques du Lac des cygnes et de La belle au bois dormant, sans perdre pour autant son avant-gardisme. Les échos des deux classiques résonnent, notamment, dans la gestuelle créée par Lock, dans la composition musicale de Gavin Bryars, Blake Hargreaves et David Lang et au sein du film réalisé par André Turpin, projeté sur trois écrans circulaires en guise d'intermèdes. Pour Lock, ce dialogue entre le passé et le présent suscite une tension qui lui sert d'inspiration :

I've always thought that memories create an interesting tension in a theater. The process by which an audience integrates and interrelates its memories onto a stage work thereby both seeing and remembering what it observes is something I've always enjoyed. Swan Lake and Sleeping Beauty are rare examples of a dancegenerated memory that has integrated itself fully into our culture, both in terms of its imagery and its music. It's unusual to find someone who doesn't have associations and recollections of parts of these ballets even if they don't know or remember when or where these memories were integrated. This lateral relationship to the source makes working with these ballets interesting and unpredictable. It is like working with a piece of living history and both influencing and being influenced by it. (4)

Selon Lock, la mémoire collective a été marquée par ces deux ballets classiques et, en partant de ce principe, il confronte dans Amjad les souvenirs qu'on a de ces ballets, qui sont souvent lourds de conventions, à la réinterprétation qu'il fait de ces classiques ${ }^{7}$.

Le titre même de sa pièce est symbolique du regard critique qu'il porte sur le monde très conventionnel de la danse classique. D'une part, l'expression arabe « Amjad » renvoie à la fois au Maroc, pays natal de Lock, et à la fascination du romantisme pour tout ce qui était considéré comme «exotique ». D'autre part, ce nom épicène dénonce la tendance qu'on avait à cette même 
époque d'attribuer aux ballets des noms de femme comme Giselle (1841), Paquita (1846) et Coppélia (1870), reflétant ainsi le culte prédominant de la féminité. En choisissant un nom qui se prête tant à un homme qu'à une femme, Lock escamote cette tradition, afin de placer le ballerin et la ballerine sur le même pied d'égalité. Par ailleurs, il s'attaque aussi à la stratégie par laquelle les ballets romantiques ont recours à l'homme en travesti, vêtu d'un costume sombre, pour évoquer la malveillance. À la différence de La belle au bois dormant de Petipa, dans laquelle seules les personnifications du mal sont revêtues de noir, dans Amjad, à une exception près, les danseurs portent tous cette couleur, éliminant ainsi la conception qu'elle serait évocatrice de la malveillance. Qui plus est, comme Ek, Lock ne fait pas de distinction entre la gestuelle des bons et celle des méchants, puisqu'il n'est plus question de raconter une histoire linéaire, mais plutôt de la déconstruire ainsi que toutes les conventions auxquelles elle renvoie. Lock réinterprète aussi la tradition rabaissante du travestissement, en créant une séquence où il met un homme sur pointes tout en le dénudant du costume féminin qu'il aurait porté auparavant. Torse nu, le ballerin retrouve la sensualité qu'on lui avait niée pendant l'époque romantique. Son corps ne représente plus une menace envers la pureté d'une beauté féminine idéalisée, d'autant plus qu'il danse son pas de deux avec un homme. Notons que dans le contexte du ballet classique, le pas de deux est traditionnellement dansé par un homme et une femme. Il constitue un duo au sein duquel le danseur est secondaire à la ballerine, la portant parfois dans ses bras, donnant ainsi l'illusion de légèreté tant recherchée pendant la période romantique. En renouvelant les conventions du pas de deux, c'est-à-dire en choisissant de mettre en vedette deux ballerins plutôt qu'un couple de sexe opposé, ainsi qu'en renversant la tradition dégradante du travestissement, Lock cherche à dénoncer la discrimination sexuelle qui a marqué le domaine du ballet classique au dix-neuvième siècle. Dans cet extrait d'Amjad, la ballerine est décentrée, à la fois sur scène (elle est placée vers l'arrière du plateau) et de manière idéologique, puisque c'est l'homme qui danse sur pointes à l'avant de la scène.

Lock utilise également l'éclairage, toujours dans cette même séquence, pour accentuer sa perception du ballet classique comme étant parfois archaïque. Contrairement au ballerin qui danse sous une lumière aux tons ambrés, produisant ainsi une impression qu'il appartient au présent, la ballerine est éclairée en bleu, couleur employée au théâtre pour évoquer le rêve ou le passé. La thématique de la désuétude du culte de la féminité est aussi reprise dans le film qui ponctue le 
spectacle, où l'on aperçoit une femme, d'une beauté frappante, dont la peau est en train de se décomposer.

Pourtant, la remise en question que fait Lock de la représentation de la notion archétypale du mal telle qu'elle est personnifiée dans les ballets romantiques par un homme en travesti est à double tranchant. En mettant un homme sur pointes, Lock présente une image précaire et fragile du ballerin, qui s'oppose diamétralement à celle qui lui était associée jadis. De la sorte, Lock oblige le public à concevoir la masculinité du danseur d'une façon nouvelle. Pour certains spectateurs, cette tâche s'avérera passionnante ; pour d'autres, qui tiennent aux conventions, ce défi sera angoissant. Le regard critique que porte Lock sur l'évocation du mal dans les ballets romantiques lui permet de remettre en valeur le ballerin, victime du dix-neuvième siècle, tout en suscitant chez certains membres de l'auditoire un malaise, comparable à celui que ressentaient les spectateurs de La belle au bois dormant à la vue de la méchante fée Carabosse mimée par un homme en travesti.

\section{Conclusion}

Tout compte fait, la dimension archétypale facilite la transposition gestuelle, visuelle et musicale d'un texte, de sorte qu'elle soit comprise par le plus grand nombre de personnes possible. Cependant, en ce qui concerne les adaptations de La belle au bois dormant de Perrault que firent Vsevolozhsky, Petipa, Ek et Lock, il n'a pas suffi de calquer les représentations du bien ou du mal telles qu'elles apparaissaient dans l'hypotexte. Chaque transposition de ces forces dichotomiques a cherché en quelque sorte à déstabiliser celle qui la précédait. Ainsi, le librettiste aussi bien que les trois chorégraphes se sont évertués à rendre tangibles et actuelles leurs évocations de la bienveillance ou de la malveillance. Pour Vsevolozhsky, ce travail se fait au moyen des procédés textuels de la reconfiguration, de la substitution, de l'élimination et de l'addition, par lesquels il unifie en un personnage les multiples personnifications que fait Perrault du mal ; explicite et amplifie la malveillance de la vieille fée qui est plutôt implicite dans le conte ; et donne un rôle plus important à la Fée des Lilas. Chez Petipa, c'est par le biais de la gestuelle, des couleurs des costumes, des leitmotive musicaux et du travestissement qu'il rend concrètes et actuelles les notions du bien et du mal dans son ballet-féerie. En revanche, pour Ek et Lock, les efforts de concrétisation et de réactualisation touchent plutôt à la notion archétypale du mal qu'à celle du bien et, pour ce faire, l'un renvoie aux inquiétudes sociales des années quatre-vingt-dix et l'autre conteste des conventions perpétuées par les ballets romantiques. 
Il s'avère que le travail effectué par le librettiste et les chorégraphes autour de l'évocation de la malveillance est un fil conducteur de cette étude, d'autant plus que leurs efforts semblent suivre une seule logique dans les exemples que nous avons analysés : quelle que soit l'époque, l'archétype est employé de sorte à ranimer dans l'esprit du lecteur ou du spectateur la réaction négative que devrait provoquer la notion du mal. Ainsi, l'usage que font Ek et Lock de cet archétype n'est pas en soi contemporain, ce sont plutôt les thématiques qu'ils décident d'explorer pour actualiser la notion du mal - comme la toxicomanie - qui le sont. La contemporanéité de l'évocation de la malveillance chez ces deux chorégraphes découle également du fait qu'ils cherchent activement à transformer le ballet de Petipa, à réinterpréter cette œuvre, notamment par le regard critique qu'ils jettent tous les deux sur les règles du ballet classique, l'un refusant d'adopter la tradition du travestissement, l'autre choisissant de réinventer celle-ci. Paradoxalement, c'est en examinant le passé, quoique de façon très critique, que ces artistes créent des œuvres dont les représentations archétypales sont fidèles à leur époque et peut-être même avant-gardistes. Qui aurait cru que le temps d'une arabesque et d'un pas de chat suffit pour bouleverser les représentations traditionnelles des notions archétypales du bien et du mal, nous obligeant ainsi à voir différemment le conte de Perrault avec chacune de ses transpositions, du ballet classique à ses manifestations contemporaines.

\section{Bibliographie}

Anthony, Sarah. « Du texte à l'arabesque : l'exemple de La belle au bois dormant ». 31 mai 2008, Colloque de l'APFUCC, UBC, Vancouver. Communication.

Burt, Ramsay. The Male Dancer : Bodies, Spectacle, Sexualities. Londres : Routledge, 2007. Canton, Katia. The Fairy Tale Revisited : A Survey of the Evolution of the Tales, from Classical Literary Interpretations to Innovative Contemporary Dance-Theatre Productions. New York : Lang, 1994.

Gard, Michael. Men Who Dance : Aesthetics, Athletics and the Art of Masculinity. New York : Lang, 2006.

Jensen, Gunilla. «Mats Ek and the Cullberg Ballet». Dancing Times (août 1997) : 993.

Jung, Carl. G. «The Concept of the Collective Unconscious ». The Collected Works of C. G. Jung. Vol. 9, tome 1. Dir. Sir Herbert Read, Michael Fordham, Gerhard Adler et William McGuire. Tr. R. F. C. Hull. Princeton : Princeton UP, 1968 [1959] : 42-53. 
Lock, Édouard. Livret de la bande sonore. Amjad, GB Records, 2008.

Midgelow, Vida. «Reworking Texts - Inverting Bodies. Mats Ek Radically Reconceives the Classics ». Dance Theatre Journal 15.2 (1999) : 4-7.

Perrault, Charles. «La belle au bois dormant». Contes. Dir. Gilbert Rouger. Paris : Garnier, 1967 [1697] : 97-107.

« Rat. » A Dictionary of Symbols. Dir. Jean Chevalier et Alain Gheerbrant. Londres : Penguin, 1996 : 788-789.

Scholl, Tim. Sleeping Beauty, a Legend in Progress. Yale UP, 2004. ProQuest Ebook

Central, https://ebookcentral.proquest.com/lib/mcgill/detail.action?docID=3419968\#.

Spångberg, Mårten. « The Psychological Dimension of Classical Dance. The Work of Swedish Choreographer Mats Ek ». Ballett International/Tanz, Aktuell 3 (mars 1996) : 40-45.

Von Franz, Marie-Louise. Shadow and Evil in Fairy Tales. Boston : Shambhala, 1995 [1974].

Wiley, Roland John. Tchaikovsky's Ballet s: Swan Lake, Sleeping Beauty, Nutcracker. Oxford : Oxford UP, 1985.

\section{Chorégraphies}

Bournonville, August. La sylphide. 1836.

Ek, Mats et al. Sleeping Beauty. Image Entertainment, 1999 [1996].

Lock, Édouard et al. Amjad. 2008 [2007].

Petipa, Marius et al. The Sleeping Beauty. Kultur, 1983 [1890].

\footnotetext{
Notes

${ }^{1}$ Le ballet-féerie « is an elaborate visual spectacle with a fairy-tale plot » (Scholl 22).

${ }^{2}$ Nous employons le terme «archétype » dans sa conceptualisation jungienne, notion qu'il définit comme «the existence of definite forms in the psyche which seem to be present always and everywhere » (42).

${ }^{3}$ Selon le Penguin Dictionary of Symbols, le rat est aussi un symbole d'avarice et de richesse.

${ }^{4}$ En revanche, à l'origine (dans les cours italiennes et françaises du Moyen-Âge et du début de la Renaissance), les hommes étaient prédominants dans le monde du ballet. C'étaient les nobles et les membres de la famille royale qui y participaient (Gard 46).

${ }^{5}$ Le Cullberg Ballet est une troupe de danse contemporaine suédoise qui compte actuellement seize danseurs et plusieurs interprètes invités. Fondé en 1967 par Birgit Cullberg, ce groupe a connu de nombreux directeurs artistiques, dont son fils Mats Ek de 1985 à 1993. Le répertoire de la troupe, qui a été présenté en Suède et à travers le monde, comprend des pièces de Cullberg et d'Ek, ainsi que d'Alvin Ailey, de Maurice Béjart, de Christopher Bruce, de Sidi Larbi Cherkaoui, de Merce Cunningham, de Flemming Flindt, d’Ulf Gadd, de Johan Inger, de Kurt Jooss, de Jiří Kylián, d'Édouard Lock, de Crystal Pite et d'Elsa-Marianne von Rosen parmi bien d'autres.

${ }^{6} \mathrm{La}$ La La Human Steps est une troupe de danse contemporaine montréalaise, qui a été fondée par Édouard Lock en 1980. Au cours de ses trente-cinq années d'existence, les membres de ce groupe ont donné des prestations au Canada
} 
et à l'étranger et ont collaboré avec des artistes de renom, comme Bette Midler, David Bowie et Frank Zappa. Malheureusement, en 2015, Lock a annoncé la dissolution de la troupe en raison de difficultés financières importantes. 7 Étant donné que la musique d'Amjad ne fait écho que très subtilement à celle de La belle au bois dormant et sans compter qu'elle renvoie aussi au Lac des cygnes de Tchaïkovski, nous ne pouvons pas faire ici d'étude comparative à partir d'une séquence musicale comme dans la section précédente de ce travail. En outre, par contraste aux chorégraphies de Petipa et d'Ek, celle de Lock n'est aucunement narrative, ce qui rend impossible une analyse par le biais de la chronologie des événements. Nous avons donc choisi de comparer l'œuvre de Petipa à celle de Lock en nous attardant sur la façon dont ce dernier réinterprète les conventions du ballet classique. 